\title{
Blood Hematocrit Still a Critical Parameter in Bioanalysis
}

Samanidou VF*

Department of Chemistry, Laboratory of Analytical Chemistry, Aristotle University of Thessaloniki, 54124 Thessaloniki, Greece

*Corresponding author: Victoria F. Samanidou, Department of Chemistry, Laboratory of Analytical Chemistry, Aristotle University of Thessaloniki, 54124 Thessaloniki, Greece, Tel: +30231997698; Fax: +302310997719; E-mail: samanidu@chem.auth.gr

Received date: January 9, 2018; Accepted date: January 12, 2018; Published date: January 19, 2018

Copyright: (c) 2018 Samanidou VF. This is an open-access article distributed under the terms of the Creative Commons Attribution License, which permits unrestricted use, distribution, and reproduction in any medium, provided the original author and source are credited.

\section{Editorial}

Recent developments in bioanalytical techniques including both sample preparation and analysis have reassured the decrease in blood sample size, a fact that is of utmost importance in cases when the available sample size is limited as for example in neonates, in postmortem samples or other populations with specific limitations e.g. anemic people, patients, elders etc. Moreover minimizing sample volume also complies with the twelve principles of green analytical chemistry.

Drugs and their metabolites can be monitored in samples of reduced volume to produce valuable and reliable results in pharmacokinetic studies, toxicokinetic studies, disease diagnoses, disease prognosis in metabolomics studies, therapeutic drug monitoring, forensics etc. Therefore microextraction techniques or micro sampling techniques have been introduced.

Dried blood spot sampling is an effective approach to this direction. Implementing dried blood microsamples in the bioanalytical workflow renders sample collection, transport, storage and processing much simpler and user friendly. It also enables the sampling in the patient's home, if necessary.

However it is known that blood hematocrit (Hct) can play a determining role in those analyses as for example in dried blood spot sampling by affecting the diameter of the derived Dried Blood Spots (DBS) sample. Hematocrit influences not only the homogeneity of sample but drug distribution as well. This is because the viscosity of blood samples which is directly related to their Hct values, may lead to non- homogenous spots since and samples with higher Hct values yield spots with smaller diameter and vice versa. Moreover taking a fixed-sized sub-punch from a high Hct DBS will contain a higher amount of blood (and consequently of analyte) than a punch taken from a low Hct DBS and this leads to a Hct-dependent assay bias. Not to mention that high Hct levels may influence the nalytes' recovery from DBS [1-10].

As it is well known the term Hematocrit refers to the fraction of blood volume that is consisted of red blood cells (erythrocytes), which comprise one of the major constituents of whole blood besides the plasma. Although plasma and serum are the preferred samples for most analytical procedures, the analysis of whole blood samples is often required as for example in post mortem cases or in cases where the shipment of the sample can be simplified and safer. In these cases the evaluation of Hct's effect on whole blood samples analysis should be taken into consideration.

To overcome this negative effect, the analysis of whole spot is recommended. However, this approach requires the accurate and precise application of a fixed volume of blood onto the filter paper using suitable pipettes or microcapillaries. But this approach is not feasible when sampling is to be carried out by non-qualified personnel as for example in patient's home.

Alternative ways to overcome the hematocrit issue involve "Volumetric absorptive microsampling" (VAMS), which is a simple technique for collecting and quantitative analysis of dried blood samples. It enables the collection of an accurate blood volume (approximately $10 \mu \mathrm{L}$ ), which is independent of the HCT of the blood, therefore no specialized devices, such as pipettes or capillaries are required. This device consists of a plastic handler with an absorbent polymeric tip, which is attached. This tip when dipped into blood, wicks up an accurate volume of blood.

Microfluidic devices consisting of a foldable support system that holds a DBS card on one side and a microfluidic plate with sized capillaries on the other have been also introduced.

Other approaches such as the application of microextraction techniques such as Solid-phase microextraction (SPME) or liquidliquid microextraction are also applied but they have been also reported to be affected by hematocrit. These problems might be overcome by using appropriate internal standards. However various analytes may be affected by Hct in a different way, therefore erythrocytes levels should be always properly considered when analyzing whole blood samples with microextraction techniques among other operation experimental conditions in the bioanalytical laboratory [11-17].

\section{References}

1. Gałuszka A, Migaszewski Z, Namieśnik J (2013) The 12 principles of green analytical chemistry and the SIGNIFICANCE mnemonic of green analytical practices. TrAC Trends Analytical Chem 50: 78-84.

2. Reyes-Garcés N, Alam N, Pawliszyn J (2018) The effect of hematocrit on solid-phase microextraction. Analytica Chimica Acta 1001: 40-50.

3. Spooner N, Olatunji A, Webbley K (2018) Investigation of the effect of blood hematocrit and lipid contents on the blood volume deposited by a disponsable dried blood spot collection device. J Pharma Biomed Anal 149: 419-424.

4. Kok MG, Fillet M (2018) Volumetric absorptive microsampling: Current advances and applications. J Pharma Biomed Anal 147: 288-296.

5. Denniff P, Spooner N (2010) The effect of hematocrit on assay bias when using DBS samples for the quantitative bioanalysis of drugs. Bioanal 2: 1385-1395.

6. O’Mara M, Hudson-Curtis B, Olson K, Yueh Y, Dunn J, et al. (2011) The effect of hematocrit and punch location on assay bias during quantitative bioanalysis of dried blood spot samples. Bioanal 3: 2335-2347.

7. De Vries R, Barfield M, van der Merbel N, Schmid B, Siethoff C, et al. (2013) The effect of hematocrit on bioanalysis of DBS: results from the EBF DBS - P. microsampling consortium. Bioanal 5: 2147-2160. 
Citation: Samanidou VF (2018) Blood Hematocrit Still a Critical Parameter in Bioanalysis. Pharm Anal Acta 9: e191. doi: $10.4172 / 2153-2435.1000 \mathrm{e} 191$

Page 2 of 2

8. De Kesel PM, Sadones N, Capiau S, Lambert WE, Stove CP (2013) Hemato-critical issues in quantitative analysis of dried blood spots: challenges and solutions. Bioanal 5: 2023-2041.

9. De Kesel PM, Lambert WE, Stove CP (2015) Does volumetric absorptive microsampling eliminate the hematocrit bias for caffeine and paraxanthine in dried blood samples? A comparative study. Analytica Chimica Acta 881: 65-73.

10. Leuthold LA, Heudi O, Déglon J, Raccuglia M, Augsburger M, et al. (2015) New microfluidic-based sampling procedure for overcoming the hematocrit problem associated with dried blood spot analysis. Anal Chem 87: 2068-2071.

11. Denniff P, Spooner N (2014) Volumetric absorptive microsampling: a dried sample collection technique for quantitative bioanalysis. Anal Chem 86 8489-8495.

12. Spooner N, Denniff P, Michielsen L, Vries RD, Ji QC, et al. (2015) A device for dried blood microsampling in quantitative bioanalysis: overcoming the issues associated blood hematocrit. Bioanal 7: 653-659.

13. Denniff P, Parry S, Dopson W, Spooner N (2015) Quantitative bioanalysis of paracetamol in rats using volumetric absorptive microsampling (VAMS) J Pharm Biomed Anal 108: 61-69.
14. Youhnovski N, Bergeron A, Furtado M, Garofolo F (2011) Pre-cut dried blood spot (PCDBS): an alternative to dried blood spot (DBS) technique to overcome hematocrit impact. Rapid Commun Mass Spectrom 25: 2951-2958.

15. Ooms B, Hempen C, Knegt L (2013) Towards unbiased dried blood spot analysis using temperature-enhanced flow-through desorption coupled online to solid phase extraction and mass spectrometry Presented at: EBF 6th Open Meeting, Barcelona, Spain.

16. De Kesel PM, Capiau S, Lambert WE, Stove CP (2014) Current strategies to cope with the hematocrit problem in dried blood spot analysis. Bioanal 6: 1871-1874.

17. Odoardi S, Anzillotti L, Strano-Rossi S (2014) Simplifying sample pretreatments: Application of dried blood spot (DBS) method to blood samples, including postmortem, for UHPLC-MS/MS analysis of drugs of abuse. Forensic Sci Intl 243: 61-67. 